\title{
PRELIMINARY STUDY OF THE SHAPE OF EURYPTERID PROSOMAS USING FOURIER ANALYSIS
}

\author{
TOLLERTON, V.P., Jr., 23 Shepherd Place, Utica, NY 13502, U.S.A.
}

Shape identification of eurypterid prosomas currently uses the ratio of prosoma length to prosoma width and the lateral angle. Because many species of eurypterids have the same prosoma shape as determined by these criteria, the method is limited in its taxonomic applications. Thompson transformation grids are of little value due to the lack of sufficient topographic reference points, even though some limited success has been achieved in its application to allometry.

Fourier analysis is a more rigorous mathematical descriptor of twodimensional shape than is simple ratios. Being independent of topographic reference points and ratios, Fourier analysis is well suited, theoretically, to the identification of prosoma shape and potential taxonomic identifications. Problems arise, though, in that Fourier analysis is sensitive to distorted specimens.

My study departs from previous methods of Fourier analysis in that the choice of the reference point from which to measure the radii is not the center of gravity, but instead is the midpoint between the eyes. My reasons for this unorthodox reference point are threefold: 1) this point is determinable on all specimens; 2) the position of the eyes is taxonomically significant (KjellesvigWaering, 1961) and 3) allows for the simultaneous identification of prosoma shape and the differentiation of taxa with similar outlines but different eye positions.

As defined by current methods, the taxa used in this study (Eurypterus remipes, E. lacustris, E. pittsfordensis, and $E$. dekayi) all have similar prosoma shapes. When specimens of the same stage of development were compared statistically, Fourier analysis was successful in the differentiation between $E$. remipes, $E$. pittsfordensis and $E$. dekayi, and between $E$. lacustris, $E$. pittsfordensis and $E$. dekayi, but not between $E$. remipes and $E$. lacustris.

The prosoma shapes as studied by Fourier analysis for both $E$. remipes and E. lacustris are also similar for all size classes examined. All size classes are dominated by the 4th harmonic. The contribution to the outline from other harmonics changes during ontogeny. The 3rd and 7th harmonics are the important components of the prosoma outline for the small size classes (classes 1-3 = maximum prosoma length of $17 \mathrm{~mm}$ ), while the 2 nd and 7 th harmonics are the important components for the larger size classes (classes 4-7). Further study involving other eurypterid taxa is in progress. 